\title{
A Novel Design of Slotted Waveguide Phased Array Antenna
}

\author{
Mohamed Elhefnawy ${ }^{1}$, Azremi Abdullah Al-Hadi ${ }^{2}$ \\ ${ }^{1}$ Dept of Electrical Engineering, Faculty of Engineering, October 6 University, Egypt \\ ${ }^{2}$ School of Computer and Communication Engineering, Universiti Malaysia Perlis, Malaysia \\ *corresponding author, E-mail: mmmel hefnawy.eng@o6u.edu.eg
}

\begin{abstract}
A novel design of phased array antenna based on a Slotted Waveguide Antenna Array (SWAA) is introduced in this paper. The CST Microwave Studio simulation results show that the S-parameters, the gain, the axial ratio, and the beamwidth of the proposed phased array antenna are affected by the value of the progressive phase shift. The simulated results of the proposed phased array antenna at different values of progressive phase demonstrate that the S-parameters for almost all ports are less than $-10 \mathrm{~dB}$ over at least $2 \%$ bandwidth, the simulated phased array antenna gain is above $17 \mathrm{~dB}$ in the frequency range from $9.5 \mathrm{GHz}$ to $9.7 \mathrm{GHz}$, the range of frequencies over which the simulated Axial Ratio (AR) is below $3 \mathrm{~dB}$ is not fixed and varied according to the selected progressive phase, the simulated beamwidth can be $6.5^{\circ}$ or narrower based on the value of the progressive phase shift.
\end{abstract}

\section{Introduction}

The waveguides are used as a transmission line in case of high power and high frequency applications. The waveguides also can work as an antenna if one end of the waveguide is opened to the space such as the horn antenna or if the walls of the waveguide have half-wavelength slots as the Slotted Waveguide Antenna Array (SWAA). The SWAA is an antenna array that consists of slots which are fed by a waveguide. The Slotted Waveguide Antenna Array (SWAA) offers many advantages such as high power handling capacity, low loss, can be used for tough environment conditions because of its metal structure, and radiation characteristics, thus they have found many applications especially in radar systems. The circularly polarized antennas are desired because it can overcome the polarization mismatch losses which are more likely to occur in the dynamic wireless environment [1]. The performance of the wireless communication systems can be improved by implementing the phased array antennas, these phased array antennas can steer the radiation pattern beam to the desired direction. This contributes to decreasing the interference and increasing the coverage distance of the wireless systems. The circularly polarized SWAA which is proposed in this paper can provide about $50 \%$ reduction in the size of the antenna array as compared to similar designs in [2-3]. Unfortunately, the previous research did not investigate the design of a phased array antenna based on circularly polarized SWAA. In this paper, the design of a phased array antenna with circularly polarized SWAA is introduced to achieve the important advantages of implementing the circular polarization, the SWAA, as well as the phased array technology. This paper is organized as follows. In Section 2 , the design of the proposed slotted waveguide phased array antenna is presented. The simulation results are given in Section 3. The conclusions are provided in Section 4.

\section{Design of the proposed slotted waveguide phased array antenna}

\subsection{Design of the proposed circularly polarized antenna array element}

The SWAA with 4-slot is considered as an antenna array element. Each antenna array element consists of total number of four slots which have the same dimensions (two slots are longitudinal and the other two slots are transversal).

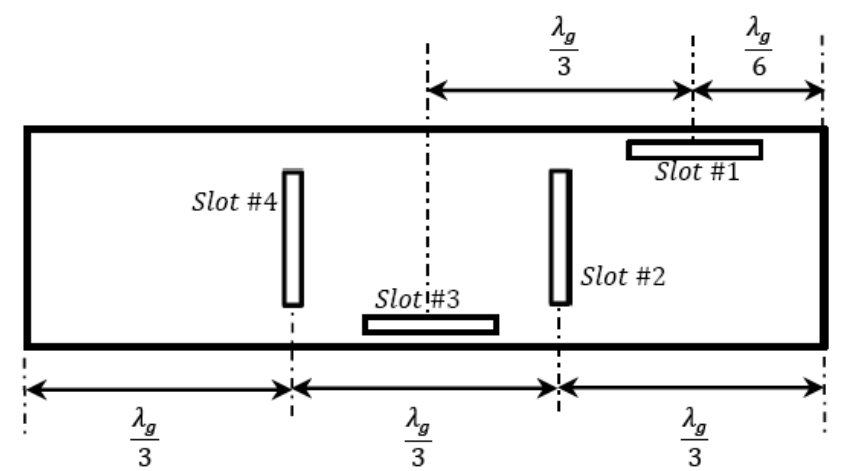

Figure 1: Structure of the resonating circularly polarized antenna array element.

Each one of these four slot antennas is obtained by cutting a narrow slot with about half wavelength long in the walls of the waveguides as shown in Fig. 1. The excitation of these slot antennas produces an electric field across the narrow dimension of each slot. The longitudinal slots in the broad wall are located at opposite sides to the central line in order to add phase shift of $(\pi)$ between the neighboring longitudinal slot fields, the distance between the neighboring longitudinal slots is one-third guide wavelength $\left(\frac{\lambda_{g}}{3}\right)$ which results in another addition of $\left(\frac{2 \pi}{3}\right)$ phase shift between the neighboring longitudinal slot fields. 
The longitudinal slot fields are added together to generate a wave which can be expressed as $-E_{L} \sin \left(\omega t+\varphi+\frac{5 \pi}{6}\right)$, where $E_{L}$ is the magnitude of the total longitudinal slot fields. The spacing between the two transversal slots is $\left(\frac{\lambda_{g}}{3}\right)$ in order to add phase shift of $\left(\frac{2 \pi}{3}\right)$ between the neighboring transversal slot fields. The fields of the neighboring transversal slots are added with each other to produce a wave which given by $E_{T} \sin \left(\omega t+\varphi-\frac{2 \pi}{3}\right)$, where $E_{T}$ is the magnitude of the total transversal slot fields. There is a phase shift of $\left(\frac{\pi}{2}\right)$ between the generated wave from the longitudinal slot fields and the wave produced by the transversal slot fields. The Right Hand Circular Polarization (RHCP) is obtained by optimizing the slot offset from the central longitudinal line in order to change the value of $E_{L}$ to be equal to the value of $E_{T}$. For the proposed antenna array element, the broad wall width of the rectangular waveguide is selected to make the cutoff frequency of $\mathrm{TE}_{10}$ mode equals $86.9 \%$ of the operating frequency at $9.6 \mathrm{GHz}$. The narrow wall width of the rectangular waveguide is selected to be half the broad wall width. The waveguide wavelength $\left(\lambda_{g}\right)$ is calculated as $\lambda_{g}=\frac{1}{\sqrt{\frac{1}{\lambda^{2}}-\frac{1}{\lambda_{c}^{2}}}}$, where $\lambda_{c}$ is

the cutoff wavelength at the operating mode, and $\lambda$ is the free space wavelength [4]. SEMI-RIGID CABLE .250 is used to feed the rectangular waveguide [5].

\subsection{Design of the proposed phased array antenna}

The elements of the proposed phased array antenna are identical and oriented in the same way, and they are fed by the same excitation amplitudes. The total radiation pattern of the phased array antenna depends on the single element pattern or the Array Factor (AF). The number of antenna array elements and the relative excitation phases can control the AF and then modify the total radiation pattern of the phased array antenna. The proposed phased array antenna is implemented by exciting each antenna array element with progressive phase relative to the preceding one. The total radiation pattern of the phased array antenna can be electronically steered to the desired direction by adjusting the value of that progressive phase. The relation between the steering angle or the desired angle of the phased array antenna radiation pattern $(\theta)$ and the progressive phase shift ( $\beta$ ) can be expressed as $\theta=\sin ^{-1}\left(\frac{\beta \lambda}{2 \pi d}\right)$, where $d$ is the centre-to-centre distance between the adjacent elements of the phased array antenna.

\section{Simulation results}

CST MWS software was used to simulate the proposed phased array antenna at operating frequency of $9.6 \mathrm{GHz}$ in case of implementing $\mathrm{TE}_{10}$ mode excitation. Table 1 indicates the dimensions of the proposed circularly polarized slotted waveguide phased array antenna with twelve elements.
Table 1: Dimensions of the proposed phased array antenna with twelve elements

\begin{tabular}{|c|c|}
\hline Parameter & $\begin{array}{l}\text { Dimensions in } \\
\mathrm{mm}\end{array}$ \\
\hline Wavelength in the waveguide $\lambda_{\mathrm{g}}$ & 63.1875 \\
\hline $\begin{array}{l}\text { Broad-wall width of the rectangular } \\
\text { waveguide }\end{array}$ & 18 \\
\hline $\begin{array}{l}\text { Narrow-wall width of the } \\
\text { rectangular waveguide }\end{array}$ & 9 \\
\hline Wavelength $\lambda$ & 31.25 \\
\hline Slot length & 15.390625 \\
\hline Slot width & 1.204 \\
\hline $\begin{array}{l}\text { Slot offset from the central } \\
\text { longitudinal line }\end{array}$ & 7.447 \\
\hline $\begin{array}{l}\text { Centre-to-centre distance between } \\
\text { the adjacent antenna array elements }\end{array}$ & $0.705 \lambda$ \\
\hline
\end{tabular}

The proposed array antenna is modeled in CST MWS. Figs. (2. a) and (2. b) show the perspective view, and the crosssectional plane view, respectively. The perspective view indicates that the proposed phased array antenna consists of twelve elements; each element has four slots (two slots are longitudinal and the other two slots are transversal). The centre-to-centre distance between the adjacent elements is $0.705 \lambda$. The rectangular waveguide for each element has cross-section dimensions of $(18 \mathrm{~mm} \times 9 \mathrm{~mm})$. The elements are separated by a metal wall, and each element is fed by the inner conductor of the coaxial cable which behaves like a monopole antenna to excite a separate $\mathrm{TE}_{10}$ mode inside each element.

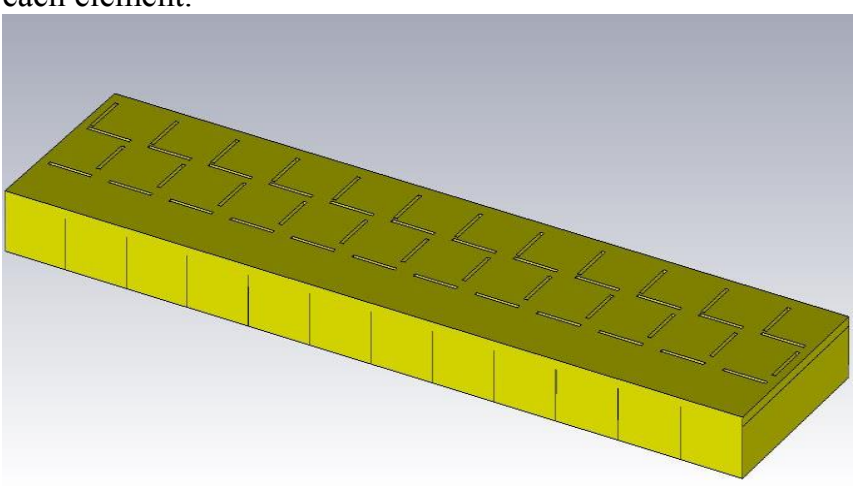

(a)

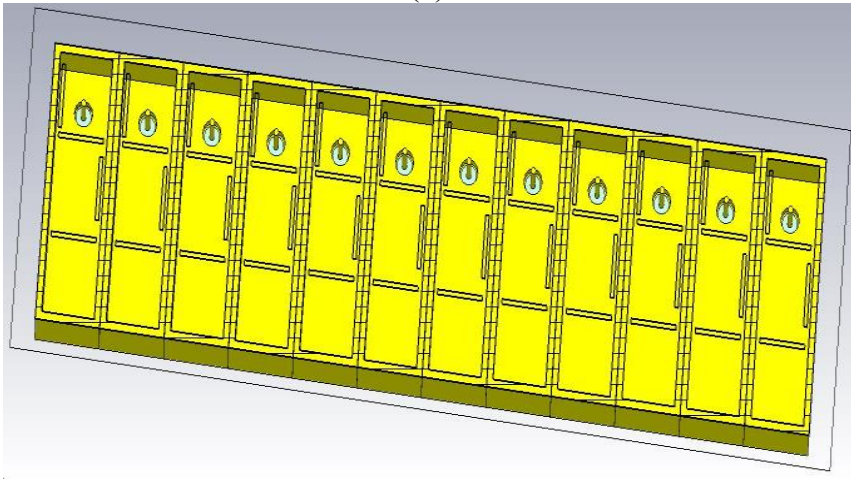

(b)

Figure 2: CST MWS model for proposed phased array antenna with twelve elements (a) Perspective view (b) Cross-sectional plane view. 
The simulated S-parameters for the proposed phased array antenna are shown in Fig. 3. The S-parameters are changed according to the selected value of the progressive phase as indicated in Figs. (3. a), (3. b), (3. c), (3. d), (3. e), (3. f), and (3. g), respectively. The S-parameters for all ports are less than $-10 \mathrm{~dB}$ in the frequency range from $9.5 \mathrm{GHz}$ to 9.7 $\mathrm{GHz}$ except at progressive phase $=90^{\circ}$ the reflection coefficient at first port is below $-8 \mathrm{~dB}$.

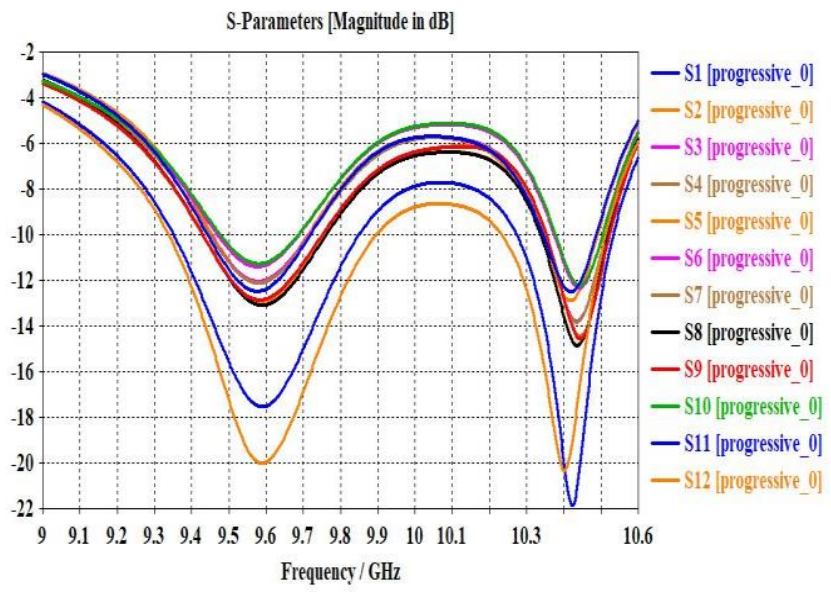

(a)

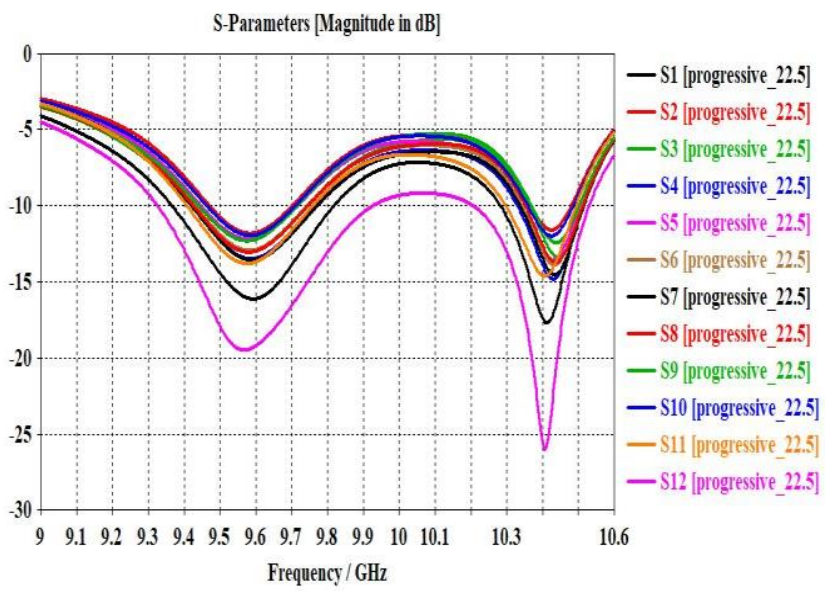

(b)

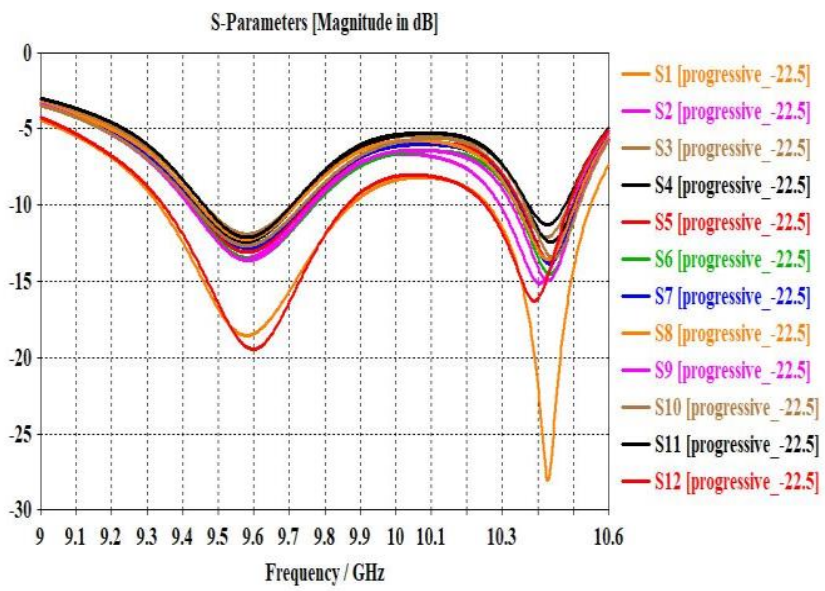

(c)

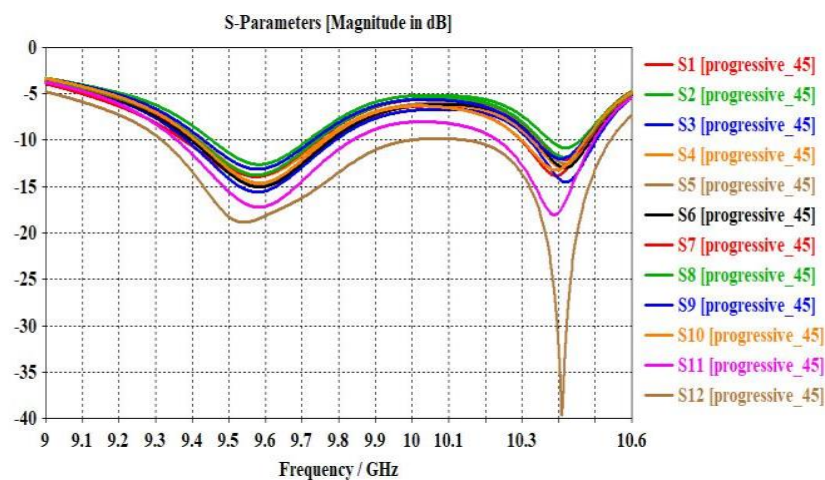

(d)

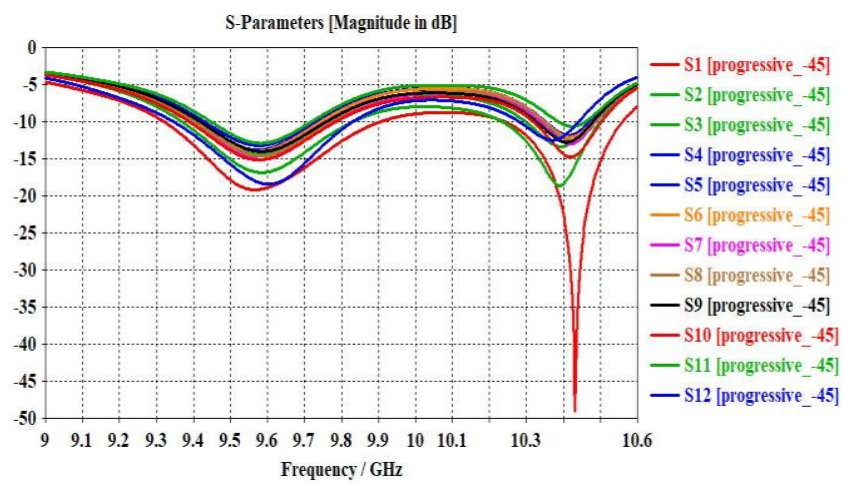

(e)

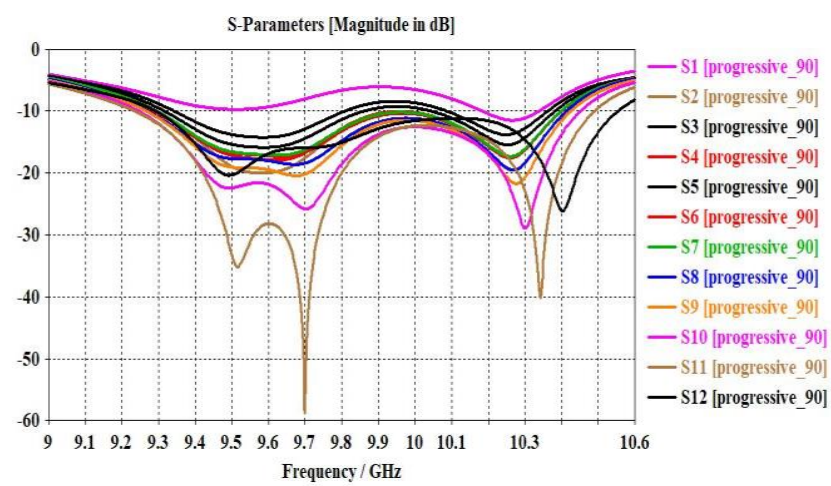

(f)

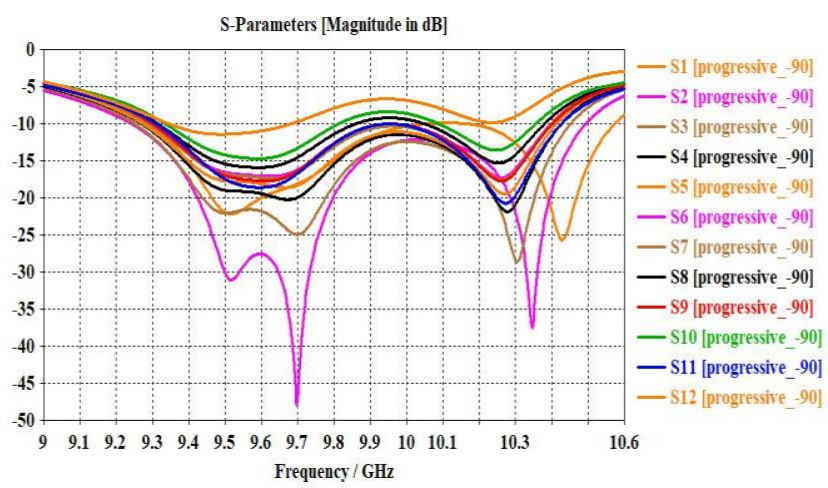

(g)

Figure 3: Simulated S-parameters for the proposed phased array antenna (a) Progressive phase shift = $0^{\circ}$ (b) Progressive phase shift $=22.5^{\circ}$ (c) Progressive phase shift $=-22.5^{\circ}$ (d) Progressive phase shift $=45^{\circ}$ (e) Progressive phase shift $=-45^{\circ}$ (f) Progressive phase shift $=$ $90^{\circ}(\mathrm{g})$ Progressive phase shift $=-90^{\circ}$. 
The two-dimensional radiation patterns of the proposed phased array antenna at different progressive phase values are shown in Fig. 4. Table 2 shows a good agreement between the calculated and the simulated radiation pattern desired angle $(\theta)$. The beamwidth is changed as the value of the progressive phase is adjusted, but the obtained beamwidth of the proposed phased array antenna is equal or less than $6.5^{\circ}$.

Table 2: Relationship between the progressive phase shift $(\beta)$ and the radiation pattern desired angle $(\theta)$

\begin{tabular}{ccc}
\hline $\begin{array}{c}\text { Progressive phase } \\
\text { shift }(\beta)\end{array}$ & $\begin{array}{c}\text { The calculated } \\
\text { desired angle of } \\
\text { the phased array } \\
\text { antenna radiation } \\
\text { pattern }(\theta)\end{array}$ & $\begin{array}{c}\text { The simulated } \\
\text { desired angle of } \\
\text { the phased array } \\
\text { antenna radiation } \\
\text { pattern }(\theta)\end{array}$ \\
\hline $0^{\circ}$ & $0^{\circ}$ & $0^{\circ}$ \\
$22.5^{\circ}$ & $5.086^{\circ}$ & $5^{\circ}$ \\
$-22.5^{\circ}$ & $-5.086^{\circ}$ & $-5^{\circ}$ \\
$45^{\circ}$ & $10.212^{\circ}$ & $10^{\circ}$ \\
$-45^{\circ}$ & $-10.212^{\circ}$ & $-10^{\circ}$ \\
$90^{\circ}$ & $20.769^{\circ}$ & $21^{\circ}$ \\
$-90^{\circ}$ & $-20.769^{\circ}$ & $-21^{\circ}$ \\
\hline
\end{tabular}

Farfield Gain Abs (Phi=0)

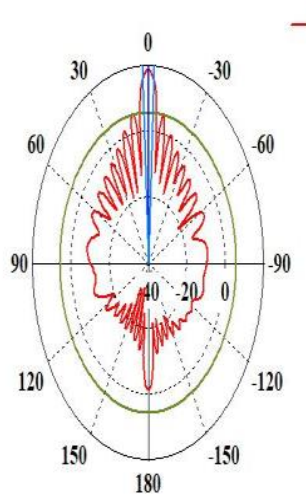

— farf...( $(\mathrm{f}=9.0)$ [progressive 0$]$

Frequency $=9$.

Main lobe magnitude $=18.6 \mathrm{~dB}$

Main lobe direction $=0.0 \mathrm{deg}$.

Angular width $(3 \mathrm{~dB})=6.0 \mathrm{deg}$.

Theta / Degree vs, dB

Side lobe level $=-12.7 \mathrm{~dB}$

(a)

Farfield Gain Abs (Phi=0)

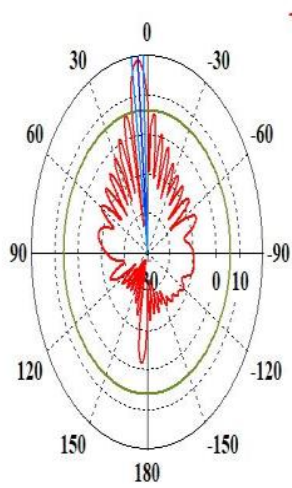

Frequency $=9.6$

Main lobe magnitude $=18.8 \mathrm{~dB}$

Main lobe direction $=\mathbf{5 . 0 \mathrm { deg }}$

Angular width $(3 \mathrm{~dB})=6.0 \mathrm{deg}$.

Theta / Degree vs. dB

Side lobe level $=-12.7 \mathrm{~dB}$

Farfield Gain Abs (Phi=0)

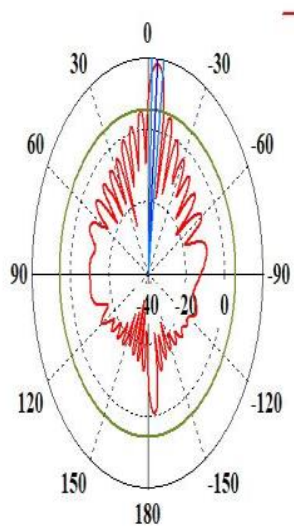

Theta / Degree rs. dB

(c)

Farfield Gain Abs (Phi=0)

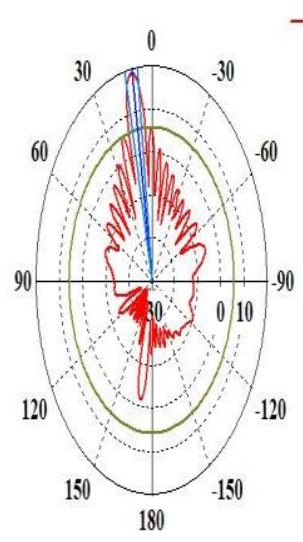

Frequency $=9.6$

Main lobe magnitude $=19 \mathrm{~dB}$

Main lobe direction $=10.0 \mathrm{deg}$.

Angular width $(3 \mathrm{~dB})=6.1 \mathrm{deg}$,

Theta / Degree rs, dB

Side lobe level $=-13.3 \mathrm{~dB}$

(d)

\section{Farfield Gain Abs (Phi=0)}

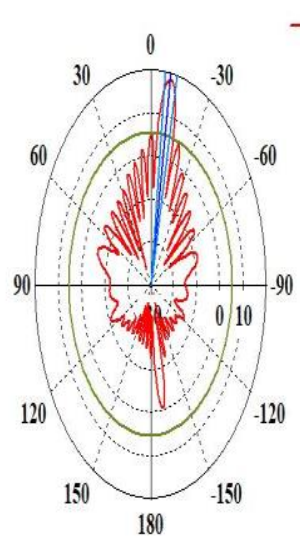

Theta / Degree vs. dB

(e)
Frequency $=9.6$

Main lobe magnitude $=18.4 \mathrm{~dB}$

Main lobe direction $=\mathbf{5 . 0} \mathrm{deg}$.

Angular width $(3 \mathrm{~dB})=6.1 \mathrm{deg}$.

Side lobe level $=-12.8 \mathrm{~dB}$

— far...( $(=9.0)$ [progressive_45]

(b) 


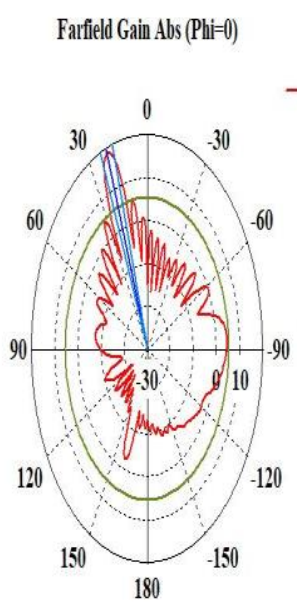

Theta / Degree rs. dB

(f)

\section{Farfield Gain Abs (Phi=0)}

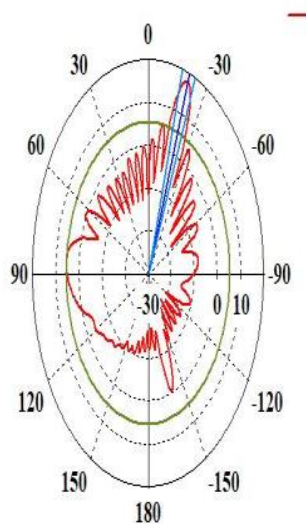

Theta / Degree rs, dB
— far...(f=9.6) [progressive 90 ]

Frequency $=9.6$

Main lobe magnitude $=18.7 \mathrm{~dB}$

Main lobe direction $=21.0 \mathrm{deg}$. Angular width $(3 \mathrm{~dB})=6.5 \mathrm{deg}$.

Side lobe level $=-13.4 \mathrm{~dB}$

(g)

Figure 4: Two-dimensional radiation patterns for the proposed phased array antenna (a) Progressive phase shift $=$ $0^{\circ}$ (b) Progressive phase shift $=22.5^{\circ}$ (c) Progressive phase shift $=-22.5^{\circ}$ (d) Progressive phase shift $=45^{\circ}$ (e) Progressive phase shift $=-45^{\circ}$ (f) Progressive phase shift $=$ $90^{\circ}(\mathrm{g})$ Progressive phase shift $=-90^{\circ}$.

Fig. 5 shows the three-dimensional radiation patterns of the proposed phased array antenna at different values of the progressive phase shift and operating frequency of $9.6 \mathrm{GHz}$. The proposed phased array antenna provides a maximum gain of $19.03 \mathrm{~dB}$ when the Progressive phase shift $=45^{\circ}$, while the minimum achieved gain is $17.87 \mathrm{~dB}$ at Progressive phase shift $=-90^{\circ}$. The obtained total efficiency of the proposed phased array antenna is above $91.8 \%$ as indicated in Table 3 which summarizes the achieved gain and total efficiency for different values of the progressive phase shift.

Table 3: The achieved gain and total efficiency for different values of progressive phase shift

\begin{tabular}{ccc}
\hline $\begin{array}{c}\text { Progressive phase } \\
\text { shift }(\beta)\end{array}$ & Gain $(\mathrm{dB})$ & Total efficiency \\
\hline $0^{\circ}$ & 18.57 & \\
$22.5^{\circ}$ & 18.79 & $91.8 \%$ \\
$-22.5^{\circ}$ & 18.42 & $92.1 \%$ \\
$45^{\circ}$ & 19.03 & $92.3 \%$ \\
$-45^{\circ}$ & 18.34 & $93.2 \%$ \\
$90^{\circ}$ & 18.7 & $93.6 \%$ \\
$-90^{\circ}$ & 17.87 & $93.2 \%$ \\
\hline
\end{tabular}

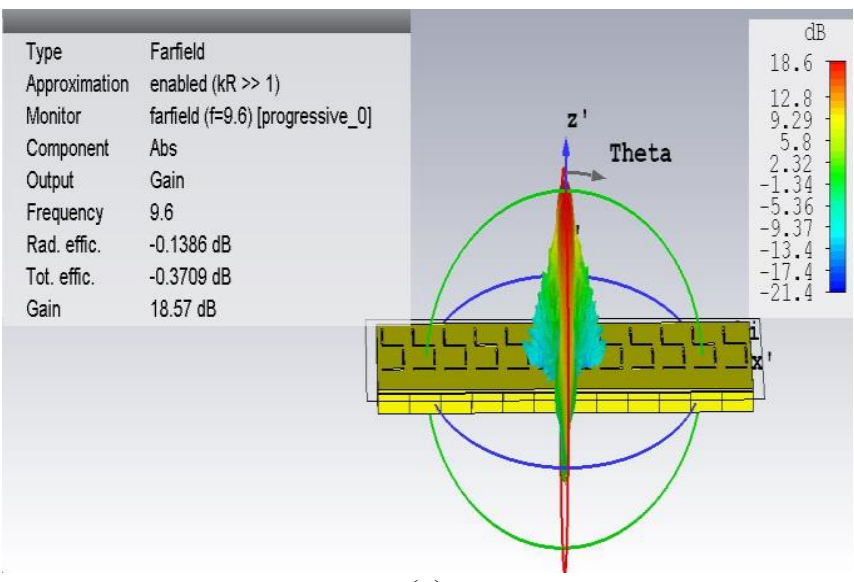

(a)

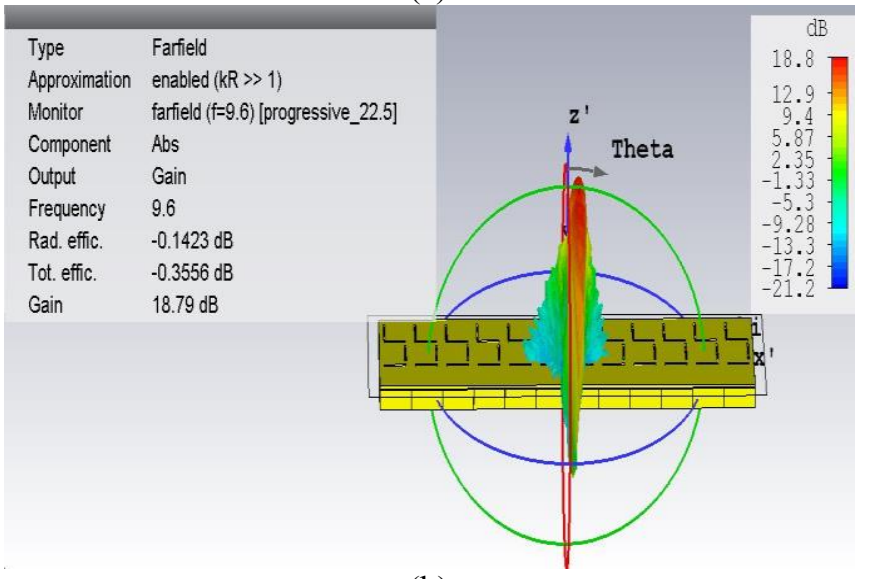

(b)

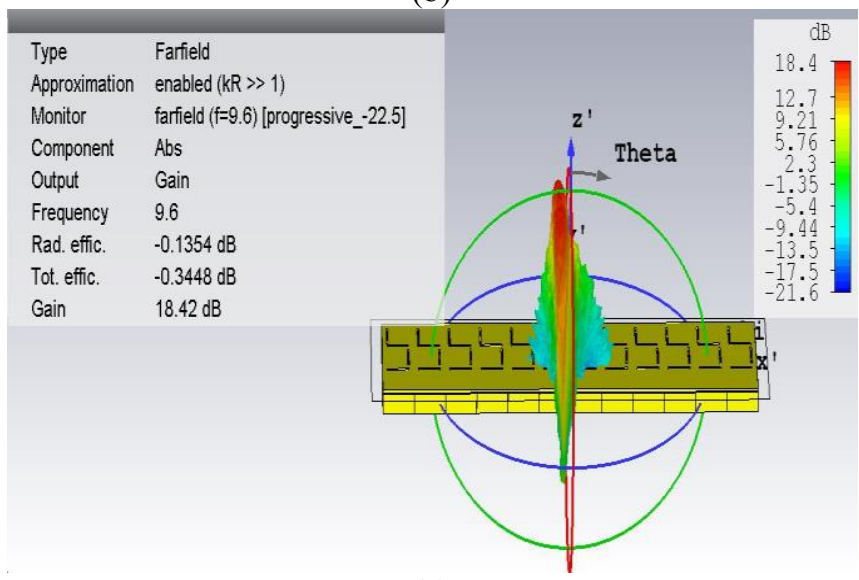

(c) 


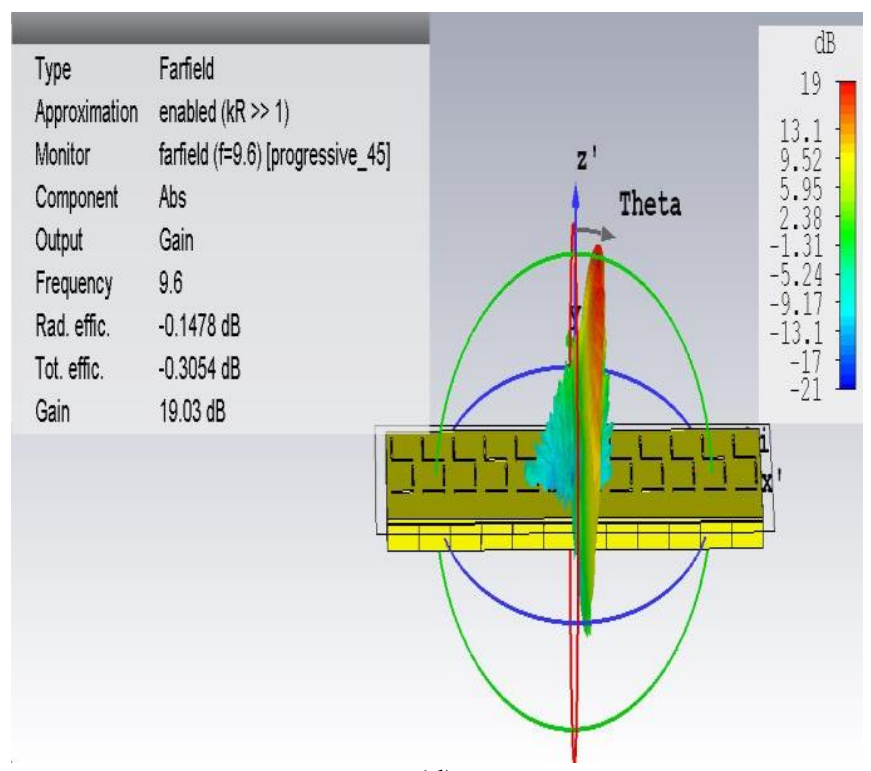

(d)

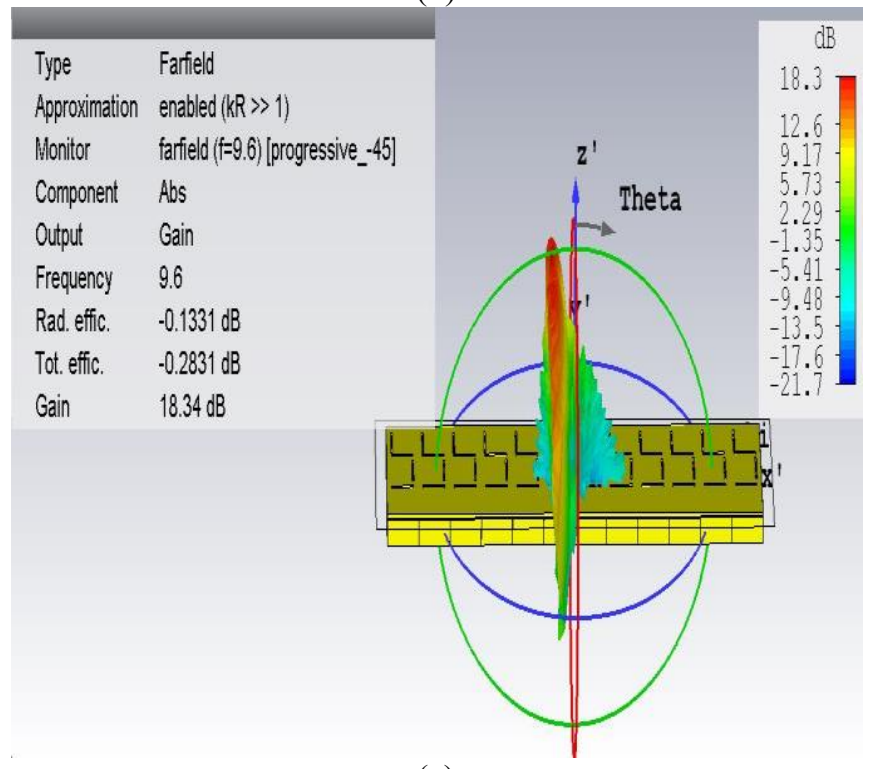

(e)

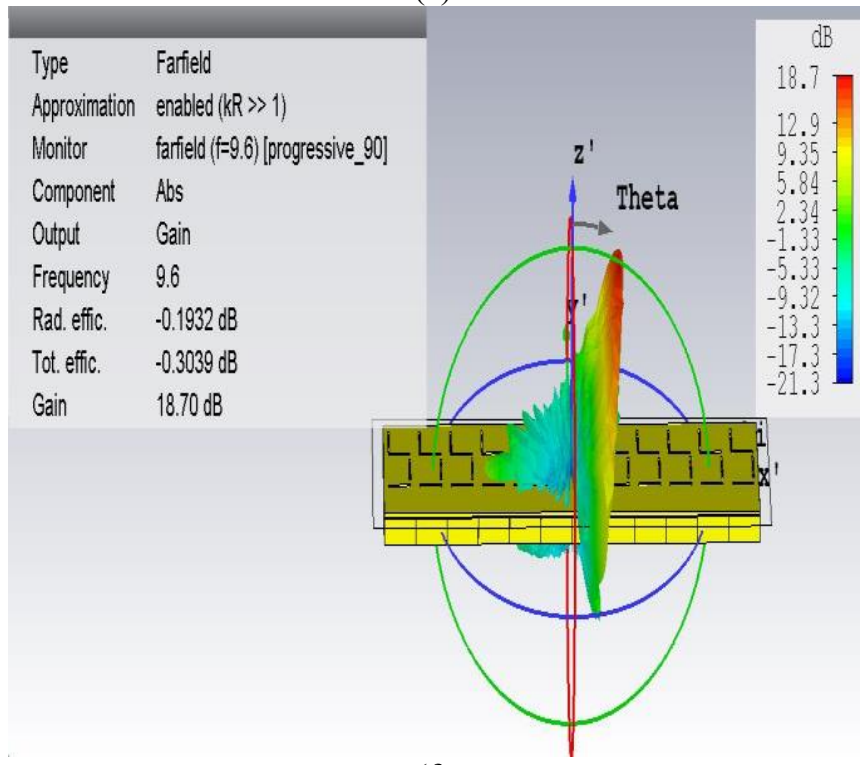

(f)

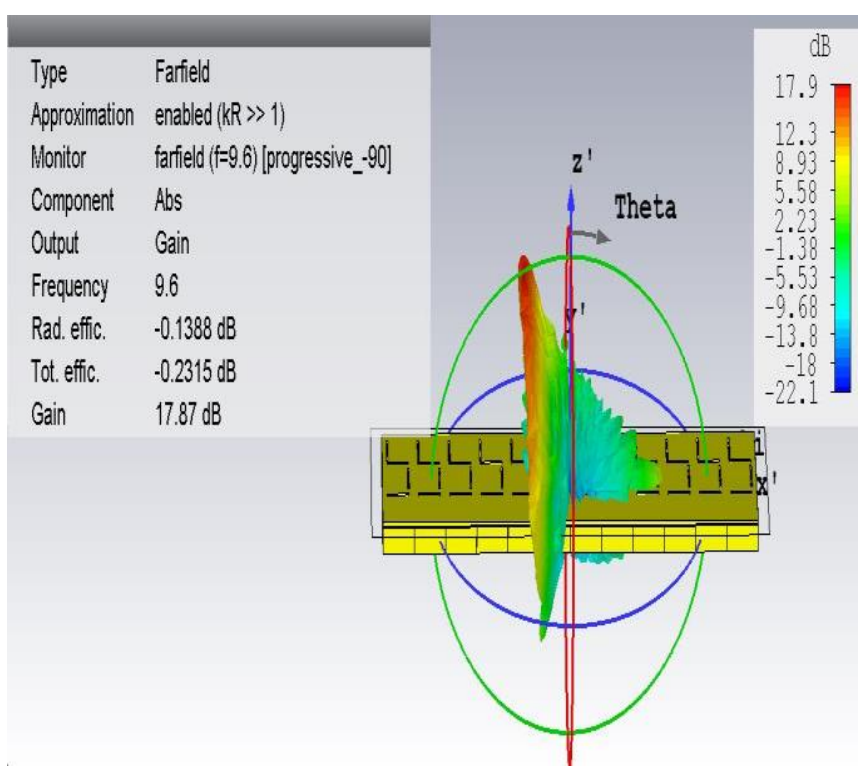

(g)

Figure 5: Three-dimensional radiation patterns for the proposed phased array antenna (a) Progressive phase shift $=$ $0^{\circ}$ (b) Progressive phase shift $=22.5^{\circ}$ (c) Progressive phase shift $=-22.5^{\circ}$ (d) Progressive phase shift $=45^{\circ}$ (e) Progressive phase shift $=-45^{\circ}$ (f) Progressive phase shift $=$ $90^{\circ}(\mathrm{g})$ Progressive phase shift $=-90^{\circ}$.

The simulated phased array antenna gain is above $17 \mathrm{~dB}$ in the frequency range from $9.5 \mathrm{GHz}$ to $9.7 \mathrm{GHz}$ as shown in Fig. 6.

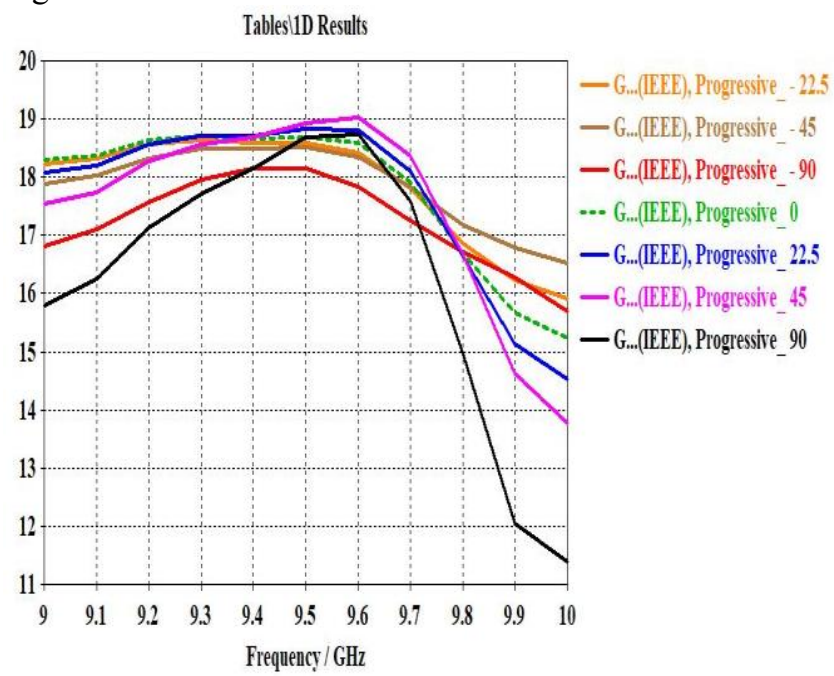

Figure 6: Simulated phased array gain versus frequency.

The range of frequencies over which the simulated Axial Ratio (AR) is below $3 \mathrm{~dB}$ is not fixed and varied according to the selected progressive phase shift as indicated in Fig. 7. Table 4 indicates that the AR is affected by the selected progressive phase shift, and the proposed phased array antenna is circularly polarized over the whole frequency range from $9.5 \mathrm{GHz}$ to $9.7 \mathrm{GHz}$ in case of Progressive phase shift $=0^{\circ}$, then the purity of circular polarization is degraded as the value of the progressive phase is changed far from $0^{\circ}$. When the progressive phase shift $=0^{\circ}$, the 
transversal slots (slot \#2 and slot \#4) provide electric fields which have the same direction for all the twelve antenna array elements; the excited electric fields across the longitudinal slot (slot $\# 1$ ) have the same direction in all antenna array elements, also the excited electric fields across the longitudinal slot (slot \#3) are in the same direction for all the twelve antenna array elements; so that a pure circular polarization can be obtained in this case because the magnitudes of the orthogonal resultant waves from the longitudinal and the transversal slot fields are almost equal to each other. On the other hand as the progressive phase shift is changed far from $0^{\circ}$, the excited electric fields across the transversal slots (slot \#2 and slot \#4) will have different directions in the antenna array elements; the direction of the excited electric fields in the longitudinal slot (slot \#1) will not be the same in all the antenna array elements, also the direction of the excited electric fields across the longitudinal slot (slot \#3) will not be the same in the twelve antenna array elements, so that the orthogonal resultant waves from the longitudinal and the transversal slot fields will not have the same magnitudes, and the purity of the circular polarization will be degraded especially when the value of the progressive phase shift is far from $0^{\circ}$ due to the increase in the rate of change in the direction of the excited electric fields across the slots of the proposed phased array antenna elements.

Table 4: The simulated AR for different values of progressive phase shift

\begin{tabular}{ccc}
\hline $\begin{array}{c}\text { Progressive } \\
\text { phase shift } \\
(\beta)\end{array}$ & $\begin{array}{c}3 \mathrm{~dB} \text { AR over the } \\
\text { frequency range from } 9.5 \\
\text { GHz to } 9.7 \mathrm{GHz}\end{array}$ & $\begin{array}{c}\text { Maximum } \\
\text { AR over the } \\
\text { frequency } \\
\text { range from } \\
9.5 \mathrm{GHz} \text { to } \\
9.7 \mathrm{GHz}\end{array}$ \\
\hline $0^{\circ}$ & $9.5 \mathrm{GHz}$ to $9.7 \mathrm{GHz}$ & $3 \mathrm{~dB}$ \\
$22.5^{\circ}$ & $9.550 \mathrm{GHz}$ to $9.7 \mathrm{GHz}$ & $4 \mathrm{~dB}$ \\
$-22.5^{\circ}$ & $9.5 \mathrm{GHz}$ to $9.636 \mathrm{GHz}$ & $5 \mathrm{~dB}$ \\
$45^{\circ}$ & $9.5 \mathrm{GHz}$ to $9.601 \mathrm{GHz}$ & $6.5 \mathrm{~dB}$ \\
$-45^{\circ}$ & $9.623 \mathrm{GHz}$ to $9.7 \mathrm{GHz}$ & $7.5 \mathrm{~dB}$ \\
$90^{\circ}$ & $3 \mathrm{~dB} \mathrm{AR}$ outside the & $15 \mathrm{~dB}$ \\
$-90^{\circ}$ & $9.5 \mathrm{GHz}$ to $9.532 \mathrm{GHz}$ & $13 \mathrm{~dB}$ \\
\hline
\end{tabular}

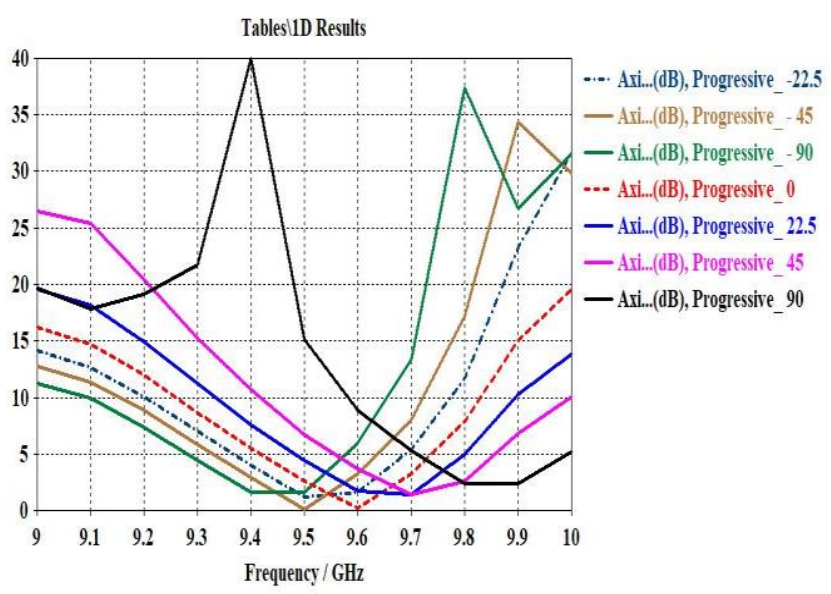

Figure 7: Simulated AR versus frequency.

\section{Conclusions}

In this paper, a proposed phased array antenna which consists of twelve slotted waveguide antenna elements is designed and simulated. The CST MWS simulation results show that the proposed phased array antenna can achieve a maximum gain of $19.03 \mathrm{~dB}$, a beamwidth equal or less than $6.5^{\circ}$ in the elevation plane, maximum total efficiency of $94.8 \%$, and S-parameters below $-10 \mathrm{~dB}$ over the frequency range from $9.5 \mathrm{GHz}$ to $9.7 \mathrm{GHz}$. The purity of the circular polarization depends on the value of the progressive phase shift. The proposed phased array antenna is a good candidate for many wireless communication systems which work in tough environment conditions, require a high power capability, small size, narrow beamwidth, and high antenna array gain.

\section{References}

[1] M. Elhefnawy and W. Ismail, "A Microstrip Antenna Array for Indoor Wireless Dynamic Environments," in IEEE Transactions on Antennas and Propagation, vol. 57, no. 12, pp. 3998-4002, Dec. 2009.

[2] Kyeong-Sik Min, J. Hirokawa, M. Ando and N. Goto, "U-shaped slots for circularly polarized slotted waveguide array," IEEE Antennas and Propagation Society International Symposium. 1995 Digest, Newport Beach, CA, USA, 1995, pp. 1434-1437 vol.3.

[3] X. 1. Jing, Z. j. Zhu, Y. z. Wang and Y. Peng, "A new kind of circular polarized slotted waveguide array antenna," 2015 16th International Conference on Electronic Packaging Technology (ICEPT), Changsha, 2015, pp. 1374-1376.

[4] S. Rozenberg and A. Yahalom, "A THz Slot Antenna Optimization using Analytical Techniques," RADIOENGINEERING, vol. 25, no. 1. Sep. 2016.

[5] https://www.radiall.com/media/files/RFCableAssemblie s\%20D1C004XEe.pdf [Accessed 7 Feb. 2018]. 